is a risk factor for ADHD that is independent of prenatal exposure to nicotine and other familial risk factors. (Mick E et al, 2002). Genes that influence the risk of alcohol and nicotine use may also influence vulnerability to ADHD. Advice regarding hazards of nicotine and alcohol exposure and monitoring of blood count and thyroid function during pregnancy are particularly important for patients with a family history of ADHD. (Millichap JG. Etiological classification of attention-deficit/hyperactivity disorder. Pediatrics 2008;121:e358-e365).

\title{
FUNCTIONAL ANATOMY OF GERSTMANN SYNDROME
}

Structural and functional neuroimaging was used to examine a common denominator for the clinical triad of Gerstmann syndrome (a selective association of acalculia, finger agnosia, left-right disorientation, and agraphia) in a study at centers in Gif-sur-Yvette and Orsay, France; and University College, I ondon, UK. None of the five neurologically healthy right-handed volunteers ( 1 female, 4 male, mean age 21 years) showed parietal overlap of cortical activation patterns from the 4 cognitive domains. Instead, these specific parietal activation patterns consistently connected to a small region of subcortical parietal white matter at a location congruent with the lesion in a documented case of Gerstmann's syndrome. Gerstmann's tetrad does not arise from damage to a shared cortical substrate in the left parietal lobe but from intraparietal disconnection after damage to a focal region of subcortical white matter. (Rusconi E, Pinel P, Eger E, et al. A disconnection account of Gerstmann syndrome: functional neuroanatomy evidence. Ann Neurol Nov 2009;66:654-662). (Respond: Dr Andreas Kleinschmidt, INSERM Unit 562, F91191 Guf-sur-Yvette, France. E-mail: kleinschmidt@cea.fr).

COMMENT. In children with learning disabilities, Gerstmann syndrome is found "forme fruste" more often than a complete Grundstorung tetrad, as proposed by Gerstmann (1940). The above disconnection account of Gerstmann syndrome with subcortical white matter pathology might explain the developmental form of the syndrome sometimes encountered in children with learning and attention disorders. A familial form of the syndrome is reported in an 11-year-old boy of normal intelligence who had a profound dyscalculia with lack of cardinal/ordinal skills acquisition, dysgraphia, right-left disorientation, and finger agnosia. Several male family members also had the complete syndrome complicated by dyslexia. At birth, the boy was hypotonic, his motor development was delayed, walking independently at 2 and $1 / 2$ years. Examination revealed gross and fine motor incoordination and inattention. Ordinal number use was compensated for by visual and verbal memory cues but cardinal number skills did not improve. (Ta'ir J et al. Brain Cogn 1997;35:184-206).

\section{TREATMENT OF ADHD AND EPILEPSY OR ABNORMAL EEG}

The comorbidity of ADHD and epilepsy is reviewed by researchers at the Universities of Rome and Chieti, Italy. In ADHD children the prevalence for epileptiform EEG discharges ranges from $5 \%$ to $60 \% ; 14 \%$ of ADHD children with epileptiform EEG abnormalities are at risk of developing seizures (Richer LP, 2002). In children with 
epilepsy the prevalence of ADHD is 30-40\% (Dunn DW, 2005), much higher than that in the general pediatric population (Schubert R, 2005). Studies differ in the number of patients, severity and type of epilepsy, and ADHD diagnosis. The types of epilepsy associated with comorbid ADHD include frontal lobe, absence, and Rolandic epilepsies. Prolonged focal epilepsy, as occurring in electrical status epilepticus during slow wave sleep, is associated with attention deficits and hyperactivity (Stores G, 1990).

Treatment of comorbid ADHD and epilepsy is a challenge because some AEDs may cause behavioral abnormalities, and stimulant medications may lower the seizure threshold. Choice of AEDs for seizure control that may improve behavior and attention in children with epilepsy and ADHD include carbamazepine or oxcarbazepine, and valproate, lamotrigine or levetiracetam. AEDs that can exacerbate behavioral disorders and should be avoided include barbiturates and topiramate. For treatment of ADHD in children with epilepsy, studies show that methylphenidate is safe in patients whose epilepsy is controlled with AEDs (Gross-Tsur V et al, 1997). Atomoxetine is recommended as an alternative to methylphenidate in some patients with ADHD and epilepsy or epileptiform discharges in the EEG (Hernandez A et al, 2005; Wernicke JF et al, 2007). Atomoxetine does not interact with AEDs while methylphenidate may increase phenytoin serum concentrations. Methylphenidate serum concentrations are lowered by carbamazepine, leading to a loss of efficacy against ADHD. (Schaller JL et al, 1999). Methylphenidate is considered the most effective and safest treatment for ADHD. (Parisi P, Moavero R, Verrotti A, Curatolo P. Attention deficit hyperactivity disorder in children with epilepsy. Brain Dev Jan 2010;32:10-16). (Respond: Dr Paolo Curatolo. E-mail: curatolo@uniroma2.it).

COMMENT. Children with ADHD are at increased risk for seizures, and children with epilepsy are at significant risk for ADHD. Management of the comorbid disorder presents a challenge because treatments for ADHD may lower seizure threshold and AEDs may exacerbate symptoms of ADHD. CNS stimulants should be avoided in children with seizures and used with caution in those with an abnormal EEG (PDR, 2009). Studies show that methylphenidate (MPH) for ADHD is safe provided that comorbid epilepsy is under control with AEDs. In patients with epileptiform EEG untreated with AEDs, the safety of MPH is less well established. Low-dose MPH does not increase the incidence of EEG epileptiform discharges. In a patient who fails to respond to low-dose stimuiant, the demonstration of epileptiform discharges by utilization of a sleep-deprived sleep EEG should aid in the choice of alternative nonstimulant medication (Millichap JJ, Stack C. Millichap JG, unpublished observations).

\section{SEIZURE DISORDERS}

\section{ACTH-INDUCED CHANGES IN IMMUNITY IN INFANTS TREATED FOR WEST SYNDROME}

The timing of vaccinations is delayed after ACTH therapy in Japanese infants, because the immune system may be compromised. To determine the duration of the effect on the immune system, researchers at Kurume University School of Medicine and other centers in Japan examined changes in immunity levels before and after ACTH 